\title{
Incidência, Distribuição Geográfica e Aspectos Ambientais das Áreas Endêmicas da Leishmaniose Visceral em Sergipe
}

\author{
Lucia Maria Sayde de Azevedo Tavares \\ Fundação Nacional de Saúde \\ Coordenação Regional de Sergipe \\ Edson Diogo Tavares \\ Empresa Brasileira de Pesquisa Agropecuária
}

\begin{abstract}
Resumo
O objetivo do presente trabalho foi avaliar a evolução da leishmaniose visceral (LV) no Estado de Sergipe, e o possível deslocamento dos focos do Sertão para o litoral nas últimas décadas, caracterizando alguns aspectos ambientais relacionados com a ocorrência dessa endemia. A metodologia consistiu da análise das notificações feitas à Fundação Nacional de Saúde entre 1972 e 1998, num total de 1.874 casos, que foram agrupados por município, por década e distribuídos nas três mesorregiões sergipanas: Sertão, Agreste e Leste (litoral). A incidência anual média da LV nas décadas de 70, 80 e 90 foi, respectivamente, de 1,96, 3,37 e 7,6. Constata-se uma grande expansão da doença em todas as regiões sem, contudo, haver deslocamento dos principais focos. Nas áreas onde se tem registrado o maior número de casos de leishmaniose visceral em Sergipe, o clima é úmido, com índices pluviométricos acima de $1.400 \mathrm{~mm}$ e baixas altitudes.
\end{abstract}

Palavras-Chave

Leishmaniose Visceral; Índices Pluviométricos; Foco Natural; Distribuição Geográfica.

\section{Summary}

The objective of this study was to evaluate the evolution of the visceral leishmaniasis $(V L)$ in Sergipe State, and a possible foci displacement from the Dry Land to the coast in the last decades, characterizing some environmental aspects related with the occurrence of the endemic disease. An analysis of all notifications send to the to National Health Foundation from 1972 to 1998 was performed. The 1.874 case identified were stratified by municipality, per decade and distributed in the three sub-areas of Sergipe: Dry Land, Transition Region and East (coast). The annual average incidence of $L V$ in the decades of 70, 80 and 90 were respectively of, 1.96, 3.37 and 7.6 per inhabitants. An expansion of this disease is verified in all areas, however there is no displacement of the main foci. In areas, where the largest number of cases of visceral leishmaniasis in Sergipe State have been registered, the climate is humid, with pluviometric indexes above 1,400 $\mathrm{mm}$ and altitude is low.

Key Words

Visceral Leishmaniasis; Pluviometric Index; Natural Foci; Geographical Distribution.

Endereço para correspondência: Lucia Sayde Tavares - Fundação Nacional de Saúde

Av. Tancredo Neves sn. Bairro Jabotiana. Aracaju-SE CEP: 49000-000

E-mail:sayde@netdados.com.br 


\section{Introdução}

Ao desenvolvimento tem sido historicamente atribuída a tarefa de melhorar a qualidade de vida da população. No entanto, no Brasil, o que se tem observado é um aumento da incidência de doenças infecciosas que estão diretamente ligadas às precárias condições de vida, e cuja disseminação está relacionada com a degradação ambiental, permitindo maior circulação dos parasitas. ${ }^{1} \mathrm{O}$ modelo de desenvolvimento atualmente adotado temse caracterizado por ausência de políticas que levem em consideração as condições em que vive a população brasileira e que promovam a organização do espaço social. Sem isso, o que tem ocorrido é a ocupação de novas áreas e a invasão do ambiente natural de forma desordenada, promovendo mudanças que levam ao desequilíbrio ecológico, expondo as populações ao risco de contrair infecções por microorganismos que circulam nos seus focos naturais. ${ }^{2}$

Desde 1934, casos humanos de leishmaniose visceral (LV) vêm sendo registrados no Estado de Sergipe. ${ }^{3}$ Em 1936, no município de Aracaju, o prof. Evandro Chagas diagnosticou o primeiro caso, em vida, da doença no Brasil. Tratava-se de um indivíduo de 16 anos, em cuja família haviam ocorrido dois óbitos, com os mesmos sintomas. ${ }^{3}$

Entre 1932 e 1957, dos 330 casos de LV identificados no Brasil, 26 eram de origem sergipana. ${ }^{4}$

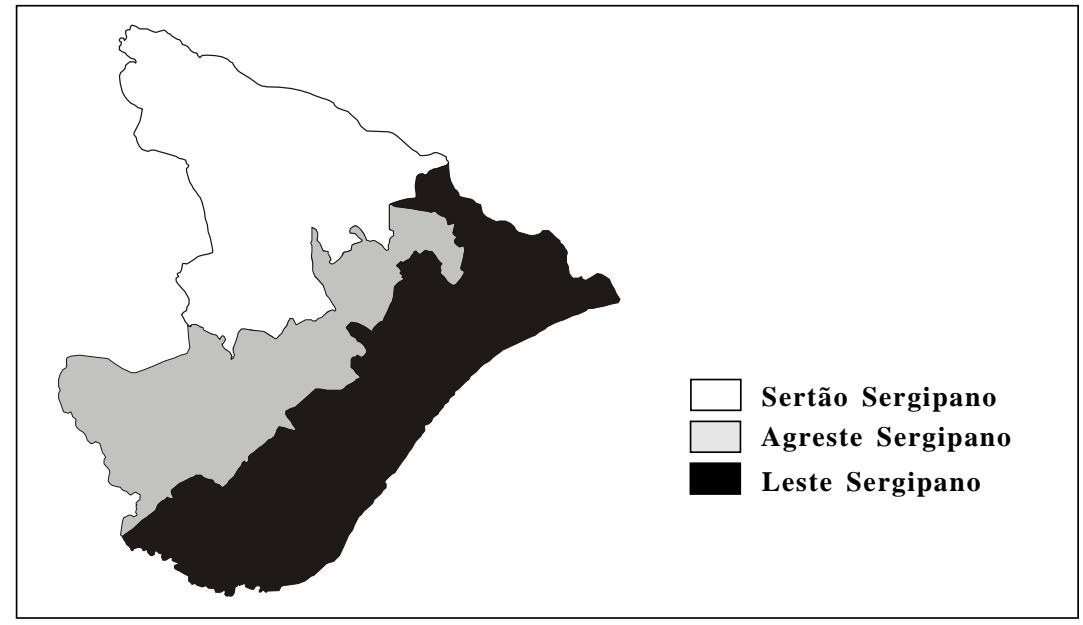

Figura 1 - Mapa das Mesorregiões de Sergipe
Devido à implantação, em 1972, da Campanha Contra a Leishmaniose, coordenada pela Superintendência de Campanhas de Saúde Pública, iniciou-se a execução dos exames de medula óssea, permitindo um maior conhecimento da distribuição e das características epidemiológicas da doença. ${ }^{5}$

Em 1983, foi feito levantamento e mapeamento das espécies flebotomínicas existentes em Sergipe, constatando-se que o inseto vetor $L$. longipalpis encontra-se disseminado em 38 municípios distribuídos em todas as regiões do Estado. ${ }^{5}$

Nos anos recentes, tem havido um aumento da ocorrência da $\mathrm{LV}$ em diversas regiões do mundo. Até início dos anos 90, no Brasil, se registrava, em média, por ano, cerca de 1.500 casos novos. ${ }^{6}$ Porém, nos últimos anos, o número de casos novos tem sido superior a 2.500 casos anuais. ${ }^{7}$

Dividido em 75 municípios, e ocupando uma área de $22.050 \mathrm{~km}^{2}$, o Estado de Sergipe corresponde a 2,8\% do território da região Nordeste do Brasil, ${ }^{8}$ região responsável por mais de $90 \%$ dos casos de LV do país. ${ }^{7}$ A população de Sergipe, projetada pelo Instituto Brasileiro de Geografia e Estatística (IBGE) para 1999, é de 1.782 .632 habitantes. ${ }^{9}$

A ocorrência de casos autóctones já foi notificada em 67 dos 75 municípios, distribuídos nas diferentes regiões do Estado, onde se encontra uma grande diversidade quanto aos aspectos ambientais.

Nos últimos anos, têm-se observado mudanças no comportamento epidemiológico da $\mathrm{LV}$, com aumento do número de casos em regiões litorâneas. Apesar disso, a doença continua sendo descrita como doença própria de clima seco com precipitação pluviométrica anual inferior a 800 $\mathrm{mm}$, e de ambiente fisiográfico composto de vales e montanhas. ${ }^{7}$

A partir de 1989, o IBGE passou a divulgar os dados do Estado de Sergipe, agregando as informações dos municípios em três mesorregiões geográficas: 
Sertão Sergipano, Agreste Sergipano e Leste Sergipano (Figura 1). ${ }^{8}$ A mesorregião Leste, relativa à área ocupada pelos municípios situados no litoral, corresponde à região de clima úmido do Estado. Para este estudo, utilizaram-se essas regiões na análise da distribuição dos casos.

O objetivo desse trabalho foi analisar a incidência, a distribuição geográfica e alguns aspectos ambientais das áreas de foco dessa endemia no Estado de Sergipe, visando melhor caracterizá-la.

\section{Metodologia}

A metodologia consistiu na análise da distribuição anual dos casos de leishmaniose visceral, por município, no período de setembro 1972 a dezembro de 1998, registrados nos arquivos da Coordenação Regional de Sergipe da Fundação Nacional de Saúde. Os dados foram agrupados por década e distribuídos pelas regiões geográficas do Estado. Foi comparada a evolução da endemia em cada período, procurando-se identificar as áreas de focos antigos e recentes, caracterizando as principais áreas endêmicas do Estado.

O registro dos casos foi feito com base no diagnóstico clínico e laboratorial. Nas décadas de 70 e 80 , todos os casos foram confirmados através do exame da medula óssea. $\mathrm{Na}$ década de 90, com a implantação do laboratório de imuno-fluorescência pela Fundação Nacional de Saúde, o método sorológico da ImunoFluorescência Indireta (IFI) passou a ser utilizado como auxílio diagnóstico em pacientes com sinais e sintomas da doença. O exame da medula óssea vem sendo realizado para a confirmação do diagnóstico em cerca de $30 \%$ dos casos.

Foram utilizados os totais pluviométricos anuais médios das últimas décadas de 45 municípios, ${ }^{10}$ visando caracterizar o clima das três mesorregiões geográficas do Estado.

Foi realizada análise da incidência média anual (número de casos novos por 100.000 habitantes), em cada uma das mesorregiões, utilizando-se dados da população residente em 1980, 1991 e população projetada para 1998 pelo IBGE. ${ }^{9}$

Tabela 1 - Número e distribuição percentual de casos humanos de leishmaniose visceral por mesorregião e por década, em Sergipe

\begin{tabular}{|c|c|c|c|c|c|c|c|c|}
\hline \multirow{3}{*}{ Mesorregião } & \multicolumn{8}{|c|}{ Década } \\
\hline & \multicolumn{2}{|c|}{70} & \multicolumn{2}{|c|}{80} & \multicolumn{2}{|c|}{ 90* } & \multicolumn{2}{|c|}{70 a $90 *$} \\
\hline & Casos & $\%$ & Casos & $\%$ & Casos & $\%$ & Casos & $\%$ \\
\hline Sertão & 30 & 16,8 & 68 & 13,5 & 140 & 11,7 & 238 & 12,7 \\
\hline Agreste & 39 & 21,8 & 49 & 9,8 & 118 & 9,9 & 206 & 11,0 \\
\hline Leste & 110 & 61,4 & 386 & 76,7 & 934 & 78,4 & 1.430 & 76,3 \\
\hline Total & 179 & 100 & 503 & 100 & 1.192 & 100 & 1.874 & 100 \\
\hline
\end{tabular}

* Dados até dezembro de 1998.

Fonte: FUNASA/SE

Tabela 2 - Municípios com maior número de casos humanos de leishmaniose visceral notificados por década, em Sergipe

\begin{tabular}{l|rrrr}
\hline \multirow{2}{*}{ Município } & \multicolumn{4}{c}{ Década } \\
\cline { 2 - 5 } & $\mathbf{7 0}$ & $\mathbf{8 0}$ & $\mathbf{9 0 *}$ & $\mathbf{7 0}$ a 90* \\
\hline Aracaju & 39 & 111 & 208 & 365 \\
Itaporanga & 14 & 51 & 150 & 215 \\
Estância & 13 & 66 & 119 & 198 \\
São Cristóvão & 11 & 32 & 99 & 142 \\
N. S. do Socorro & 6 & 21 & 68 & 95 \\
Japaratuba & 6 & 21 & 63 & 90 \\
Areia Branca & 14 & 12 & 41 & 67 \\
Japoatã & 1 & 9 & 53 & 63 \\
\hline
\end{tabular}

* Dados até dezembro de 1998.

onte: FUNASA/SE

\section{Resultados e Discussões}

No período de 1972 a 1998 foram registrados 1.874 casos de LV no Estado de Sergipe, distribuídos em 67 municípios.

Até o ano de 1979 (década de 70), ocorreram 179 casos, provenientes de 37 municípios. Os municípios com maior número de casos, com exceção do município de Areia Branca, que está situado na região Agreste, estão situados na região Leste, litoral do Estado, evidenciando que desde a década de 70 a 
doença estava associada principalmente à região de clima úmido (Tabelas 1 e 2).

$\mathrm{Na}$ década de 80 , o número de registros se elevou para 503 e na década de 90 (até dezembro de 1998) foram registrados 1.192 casos, evidenciando a grande expansão da endemia. Entre 1980 e 1989, a doença foi registrada em 47 municípios e, nos anos 90, em 65 municípios. Constata-se que a doença vem sendo notificada em um número crescente de municípios sem deixar de ocorrer nos municípios onde se registraram os primeiros casos (Tabelas 1 e 2).

Até 1993, eram registrados no melhoria do sistema de notificação. ${ }^{22}$ Além desses fatores, é importante considerar que assim como ocorreu em Sergipe, houve a implantação, em vários outros Estados, de laboratórios para a realização de exames sorológicos para LV, o que provavelmente contribuiu para o diagnóstico de um maior número de casos.

Ao analisarmos a distribuição dos casos de LV entre as três mesorregiões geográficas, observamos que, em Sergipe, a região Leste (região litorânea) sempre se destacou das demais regiões em número de casos da doença (Figura 2).

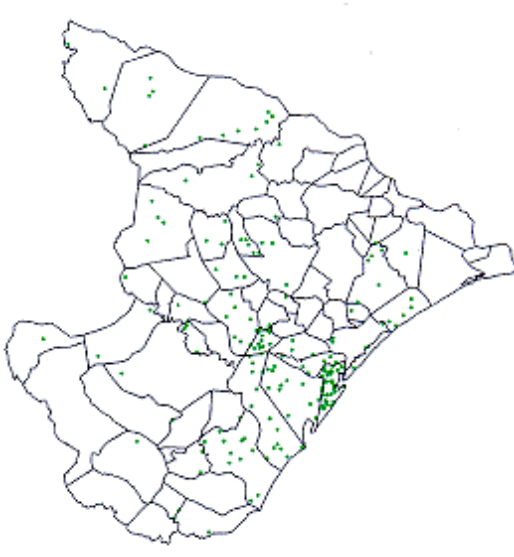

Década de 70

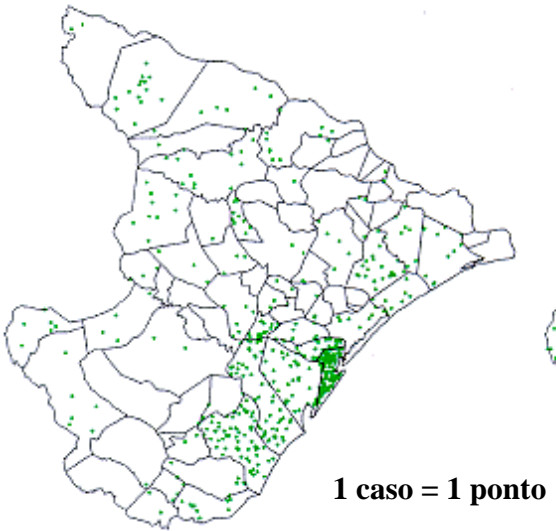

Década de 80

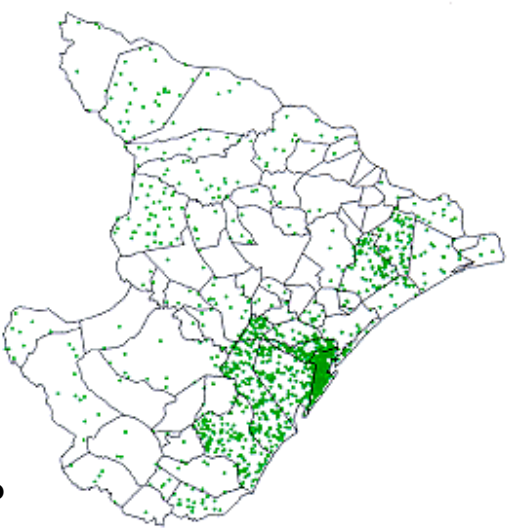

Década de 90

Figura 2 - Mapa da distribuição geográfica dos casos de leishmaniose visceral por município de Sergipe nas décadas de 70, 80 e 90.

Estado de Sergipe em média 80 casos novos por ano, mas, a partir de 1994, houve um aumento significativo do número de casos, sendo registrados 203 casos novos. No ano de 1995 foi registrado o maior número de casos de toda a história da doença em Sergipe, 266 casos distribuídos em 137 localidades de 34 municípios. ${ }^{11}$ Em 1996 foram registrados 190 casos. Nos últimos dois anos tem-se observado uma redução no total de casos novos ocorrendo 109 casos em 1997 e 94 casos em 1998.

O aumento do número de casos na década de 90 ocorreu em vários Estados do Nordeste e foi explicado tanto pelo incremento real dos casos como pela
Observa-se que nas três mesorregiões de Sergipe vem ocorrendo aumento na incidência da doença nas últimas décadas. Mesmo sendo a região Leste a que mais cresceu em termos demográficos, tendo a sua população quase que dobrado entre 1970 e 1998 , esta região apresentou o maior aumento na incidência média por ano de casos novos, passando a incidência de 1,98 nos anos 70 , para 8,87 na década de 90 , um aumento de $448 \%$ na incidência da doença (Tabela 3).

Mesmo tendo havido aumento no número total de casos nas regiões do Sertão e do Agreste, proporcionalmente, essas regiões vêm apresentando uma 
redução em relação ao total dos casos no Estado. Na década de 70 constata-se que no Sertão e no Agreste haviam ocorrido $38,6 \%$ do total de casos, e que nos anos 90 os casos dessas regiões representaram somente $21,6 \%$ do total de casos (Tabela 1).

A média dos índices pluviométricos anuais dos municípios do Estado de Sergipe que apresentaram casos humanos de LV localizados no Sertão sergipano variaram de $488 \mathrm{~mm}$ (Canindé do São Francisco) a 1.087 mm (Monte Alegre de Sergipe); na região do Agreste, de $764 \mathrm{~mm}$ (Tobias Barreto) a $1.350 \mathrm{~mm}$ (Malhador); e na região Leste, de 816 mm (Propriá) a 1.960 mm (Santa Luzia do Itanhi).

A precipitação média anual dos municípios com maior número de casos foi a seguinte: Aracaju $1.577 \mathrm{~mm}$, Itaporanga $1.463 \mathrm{~mm}$, Estância $1.469 \mathrm{~mm}$ e São Cristovão $1.491 \mathrm{~mm} .{ }^{10}$ Portanto, em Sergipe o clima das principais áreas de foco da endemia é úmido, com índices pluviométricos superiores a $1400 \mathrm{~mm}$.

A incidência da LV no Estado de Sergipe vem aumentando nas últimas décadas em todas as suas regiões, desde o Sertão, passando pelo Agreste, até a região Leste, porém de forma mais acentuada nessa última.

Os municípios que têm apresentado o maior número de casos são os mesmos desde os primeiros registros da doença. A análise dos dados sugere que a ocupação das áreas de antigos focos por uma população crescente, com o conseqüente aumento dos suscetíveis, pode ser a causa do aumento do número de casos. Mesmo havendo a expansão da endemia no Estado, não se observou o deslocamento da doença do Sertão para a região litorânea. Portanto, a doença não se caracterizou como sendo procedente das regiões de clima semi-árido como ocorreu em outras regiões do Nordeste. ${ }^{13}$
Em Sergipe observamos que, mesmo ocorrendo casos isolados em vários pontos do Estado com aspectos ambientais distintos, os focos mais antigos da doença e as principais áreas endêmicas ficam na região Leste, onde o clima é úmido, apresentando índices pluviométricos superiores a $1.400 \mathrm{~mm}$, e onde as altitudes são próximas do nível do mar.

\section{Bibliografia}

1. Sabroza PC, Toledo LM de, Osanai CHA. Organização do Espaço e os Processos Endêmico-Epidêmicos. In: Leal MC (org.) Saúde, Ambiente e Desenvolvimento. Vol II. Rio de Janeiro: Hucitec-Abrasco; 1993.

2. Mello DA. Parasitic diseases in Brazil and the role of wild mammals: an analysis based on leishmaniasis, Chagas disease and schistosomiasis mansoni. Ciência e Cultura 1991; 43(4):274-278.

3. Chagas E. Primeira verificação em indivíduo vivo da leishmaniose visceral no Brasil. Brasil-Médico 1936; 11(11): 221-222.

4. Silva JR da. Leishmaniose Visceral (Calazar) [Tese de professor catedrático]. Rio de Janeiro: Faculdade Nacional de Medicina. Universidade do Brasil; 1957.

5. Aguiar CP. Relatório Técnico da Campanha Contra a Leishmaniose. Ministério da Saúde/SUCAM, Aracaju, 1983. (mímeo).

6. Marzochi MC de A, Marzochi KBF. 
Tegumentary and visceral leihmaniases in: Brazil - emerging anthropozoonosis 1994; Supl.2: 359375 .

7. BRASIL, Ministério da Saúde. Fundação Nacional de Saúde. Controle, diagnóstico e tratamento da Leishmaniose Visceral (Calazar), 1996.

8. Diniz JAF, França VLA. Atlas SócioEconômico de Sergipe. [disquetes]. Aracaju: UFS; 1996. 4 disquetes.

9. Anuário Estatístico de Sergipe. Vol.18. Aracajú: SEPLANTEC/ SUPES; 1996.

10.Pinto JES de S. Os reflexos da seca no estado de Sergipe [Tese de Doutorado]. Rio Claro: UNESP; 1997.

11. Tavares LMS de A. Plano Operativo Técnico-Administrativo do Programa de Controle da Leishmaniose Visceral. Fundação Nacional de Saúde, Aracaju, 1996. (mímeo).

12. Monteiro PS, Lacerda MM, Arias JR. Controle da leishmaniose visceral no Brasil. Revista da Sociedade Brasileira de Medicina Tropical 1994; 27 (supl. III): 67-72.

13. Costa CHN, Pereira HF, Araújo MV. Epidemia de de leismaniose visceral no estado do Piauí, Brasil, 19801986. Revista de Saúde Pública 1990; 24(5): 361-372. 